\title{
On recursive hyperbolic functions in Fibonacci-Lucas sense
}

\author{
Ahmet Daşdemir*1 (D), Tuncay Deniz Şentürk ${ }^{2}$ (D), Zafer Ünal ${ }^{1}$ (D) \\ ${ }^{1}$ Department of Mathematics, Faculty of Arts and Sciences, Kastamonu University, 37150, Kastamonu, \\ Turkey \\ ${ }^{2}$ Department of Mathematics, Institute of Science and Technology, Kastamonu University, 37150, \\ Kastamonu, Turkey
}

\begin{abstract}
The hyperbolic Fibonacci and hyperbolic Lucas functions have been introduced before and have been improved to functions of the symmetrical form. In this paper, we generalize the mentioned definitions, which will be called Horadam hyperbolic sine function $(H S F)$ and Horadam hyperbolic cosine function $(H C F)$. Further, we present many identities and hyperbolic properties of our new definitions.
\end{abstract}

Mathematics Subject Classification (2010). 03D20, 11B37

Keywords. Horadam hyperbolic function, Horadam spiral, quasi-sine Horadam function, Honsberger formula, Vajda's identity

\section{Introduction}

The Fibonacci sequence is defined as

$$
F_{n+2}=F_{n+1}+F_{n} \text { for } n \geqslant 0
$$

with the initial terms $F_{0}=0$ and $F_{1}=1$. The Lucas sequence satisfies the recurrence relation in (1.1) but with the initial conditions $L_{0}=2$ and $L_{1}=1$. The Binet's formulas of the Fibonacci and Lucas numbers are

$$
F_{n}=\frac{\alpha^{n}-(-1)^{n} \alpha^{-n}}{\sqrt{5}}
$$

and

$$
L_{n}=\alpha^{n}+(-1)^{n} \alpha^{-n},
$$

respectively. Here, $\alpha$ is the golden ratio; see [9] for more detailed information on the subject.

In the current literature, there are many various generalizations of the Fibonacci and Lucas sequences. For example, in [5], Horadam considered a generalized form of the classic

\footnotetext{
*Corresponding Author.

Email addresses: ahmetdasdemir37@gmail.com (A. Daşdemir), tuncaydenizsenturk@gmail.com (T.D. Şentürk), zunal@kastamonu.edu.tr (Z. Ünal)

Received: 06.03.2019; Accepted: 24.03.2020
} 
Fibonacci numbers, changing the initial terms $F_{0}=0$ and $F_{1}=1$ to $a$ and $b$, respectively. Then in [6], Horadam defined the second-order linear recurrence sequence $w_{n}(a, b ; p, q)$ :

$$
w_{n}=p w_{n-1}-q w_{n-2}
$$

with $w_{0}=a$ and $w_{1}=b$. In addition, the author also gave the Binet's formula for the Horadam sequence as

$$
w_{n}=\frac{A \alpha^{n}-B \beta^{n}}{\alpha-\beta},
$$

where $\alpha$ and $\beta$ are the roots of $x^{2}-p x+q=0, A=b-a \beta$, and $B=b-a \alpha$. In [7] and [8], the Horadam presented some applications and geometrical interpretations of the Horadam sequence, including the Pythagorean property. This generalizes many sequences of integers; e.g., the Fibonacci, Lucas, Pell, Pell-Lucas, Jacobsthal, Jacobsthal-Lucas, generalized Fibonacci, and generalized Lucas sequences.

We can summarize the generalization processes as follows. Each term of the sequence is defined as a linear combination of the preceding two terms; the initial conditions of the sequence can be real or complex variables in addition to the linear combination; and each term is a sum of $k$ preceding terms with $k$ initial conditions. However, in [17], Stakhov and Tkachenko presented one of the most interesting approaches on the subject except for these procedures. Considering the Binet's formulas of the Fibonacci and Lucas sequences, the authors introduced new concepts that are called hyperbolic Fibonacci and hyperbolic Lucas functions. Furthermore, in [14], Stakhov and Rozin improved these approaches to more simple forms, which are called the symmetric hyperbolic Fibonacci and symmetric hyperbolic Lucas functions, as

Symmetric hyperbolic Fibonacci sine function: $s F s(x)=\frac{\alpha^{x}-\alpha^{-x}}{\sqrt{5}}$,

Symmetric hyperbolic Fibonacci cosine function: $c F s(x)=\frac{\alpha^{x}+\alpha^{-x}}{\sqrt{5}}$,

Symmetric hyperbolic Lucas sine function: $s L s(x)=\alpha^{x}-\alpha^{-x}$,

and

Symmetric hyperbolic Lucas cosine function: $c L s(x)=\alpha^{x}+\alpha^{-x}$,

where $x$ is any real number. The monograph in [12] displays a detailed review. It should be noted that $s F s(2 k)=F_{2 k}, c L s(2 k)=L_{2 k}, c F s(2 k+1)=F_{2 k+1}$, and $s L s(2 k+1)=$ $L_{2 k+1}$ can be written for $k \in Z$. In [15], Stakhov and Rozin extended the definitions given in (1.6)-(1.9) to the special second-degree function to be called "the quasi-sine Fibonacci function" as follows:

Quasi-sine Fibonacci function: $F F(x)=\frac{\alpha^{x}-\cos (\pi x) \alpha^{-x}}{\sqrt{5}}$.

In [3], Falcón and Plaza introduced the $k$-Fibonacci sequence as

$$
F_{k, 0}=0, F_{k, 1}=1 \text {, and } F_{k, n+1}=k F_{k, n}+F_{k, n-1} \text { for } n \geqslant 1,
$$

where $k$ is any integer number. In addition, the authors showed that Eq. (1.11) satisfies the Binet-like formula

$$
F_{k, n}=\frac{r_{1}^{n}-r_{2}^{n}}{r_{1}-r_{2}}
$$

where $r_{1}$ and $r_{2}$ are the positive and negative roots of the characteristic equation $r^{2}=$ $k r+1$, respectively. Inspired by the definitions in Eqs. (1.6)-(1.10), in [4], Falcón and 
Plaza gave new generalizations of the quasi-sine and hyperbolic $k$-Fibonacci functions as

$k-$ Fibonacci hyperbolic sine function: $s F_{k} h(x)=\frac{\sigma^{x}-\sigma^{-x}}{\sigma+\sigma^{-1}}$,

$k$ - Fibonacci hyperbolic cosine function: $c F_{k} h(x)=\frac{\sigma^{x}+\sigma^{-x}}{\sigma+\sigma^{-1}}$,

and

Quasi-sine $k$ - Fibonacci function: $c F_{k} h(x)=\frac{\sigma^{x}-\cos (\pi x) \sigma^{-x}}{\sigma+\sigma^{-1}}$,

where $k$ is any positive real number and $\sigma$ is the positive root of $\sigma^{2}=k \sigma+1$. Note that the above-mentioned functions have many applications in mathematics and physics. In particular, the hyperbolic Fibonacci and Lucas functions are encountered for solving some partial differential equations numerically. The papers in [1]-[2], [10]-[11], [12], and [16]-[13] contain their some applications.

It is clear that the definitions in (1.6)-(1.15) depend, except for the real variable $x$, either on certain fixed real numbers or to a real number that is to be determined beforehand, e.g. $\alpha$ or $k$. In this study, we remove these restrictions. To do this, we present new definitions that generalize the hyperbolic-type functions given in (1.6)-(1.15). Further, we give some important results, including Catalan-type identity and Honsberger formula.

\section{Main results}

Let $f(x)$ be an arbitrary continuous function of real number $x$. We now consider the second-order equation

$$
\lambda^{2}=f(x) \lambda+1 .
$$

Hence, Eq. (2.1) has the following distinct two roots

$$
\lambda_{1}=\alpha=\frac{f(x)+\sqrt{f^{2}(x)+4}}{2} \text { and } \lambda_{2}=\beta=\frac{f(x)-\sqrt{f^{2}(x)+4}}{2} .
$$

Here, we can write

$$
\alpha+\beta=f(x), \alpha \beta=-1, \quad \alpha-\beta=\Delta=\sqrt{f^{2}(x)+4} .
$$

We can start with the following definition that is our fundamental result.

Definition 2.1. Let $f(x), a(x)$, and $b(x)$ be any continuous functions. Then, a Horadam hyperbolic sine function $(H S F)$ and a Horadam hyperbolic cosine function $(H C F)$ are respectively defined as

$$
\mathcal{H}_{s}(a, b, f, x)=\mathcal{H}_{s}(x)=\frac{A \alpha^{x}-B \alpha^{-x}}{\Delta}
$$

and

$$
\mathcal{H}_{c}(a, b, f, x)=\mathcal{H}_{c}(x)=\frac{A \alpha^{x}+B \alpha^{-x}}{\Delta},
$$

where $\Delta=\sqrt{f^{2}(x)+4}, A=b(x)+a(x) \alpha^{-1}$ and $B=b(x)-a(x) \alpha$.

This generalizes Fibonacci hyperbolic functions popularized by Stakhov and Tkachenko [17] and improved by Stakhov and Rozin [14]. Table 1 displays some special cases for our definitions. Here, the symbol $(*)$ means that the corresponding hyperbolic functions have yet to be studied.

In working with these functions, it is useful to consider the following special cases:

$$
\mathcal{H}_{s}(0,1, f, x)=\mathcal{U}_{s}(x)=\frac{\alpha^{x}-\alpha^{-x}}{\Delta},
$$


Table 1. Special cases for Horadam hyperbolic functions

\begin{tabular}{|c|c|c|c|c|c|}
\hline \multirow{3}{*}{ Horadam HF } & \multicolumn{2}{|c|}{ Symbols } & \multirow{3}{*}{$a(x)$} & \multirow{3}{*}{$b(x)$} & \multirow{3}{*}{$f(x)$} \\
\hline & Sine & Cosine & & & \\
\hline & $\mathcal{H}_{s}(x)$ & $\mathcal{H}_{c}(x)$ & & & \\
\hline Generalized Fibonacci HF & $\mathcal{U}_{s}(x, f(x))$ & $\mathcal{U}_{c}(x, f(x))$ & 0 & 1 & $f(x)$ \\
\hline Generalized Lucas HF & $\bar{V}_{c}(x, f(x))$ & $\bar{V}_{s}(x, f(x))$ & 2 & $f(x)$ & $f(x)$ \\
\hline Fibonacci HF $[14,17]$ & $s F s(x)$ & $c F s(x)$ & 0 & 1 & 1 \\
\hline Lucas HF $[14,17]$ & $c L s(x)$ & $s L s(x)$ & 2 & 1 & 1 \\
\hline$k$-Fibonacci HF [4] & $s F_{k} h(x)$ & $c F_{k} h(x)$ & 0 & 1 & $k$ \\
\hline Pell HF* & $s P s(x)$ & $c P s(x)$ & 0 & 1 & 2 \\
\hline Pell-Lucas HF* & $c Q s(x)$ & $s Q s(x)$ & 2 & 2 & 2 \\
\hline Modified Pell HF* & $c R s(x)$ & $s R s(x)$ & 1 & 1 & 2 \\
\hline
\end{tabular}

$$
\begin{aligned}
& \mathcal{H}_{c}(0,1, f, x)=\mathcal{U}_{c}(x)=\frac{\alpha^{x}+\alpha^{-x}}{\Delta}, \\
& \mathcal{H}_{s}(2, f, f, x)=\mathcal{V}_{c}(x)=\alpha^{x}+\alpha^{-x}, \\
& \mathcal{H}_{c}(2, f, f, x)=\mathcal{V}_{s}(x)=\alpha^{x}-\alpha^{-x} .
\end{aligned}
$$

We will call the sequences $\mathcal{U}_{c}(x), \mathcal{U}_{s}(x), \mathcal{V}_{c}(x)$, and $\mathcal{V}_{s}(x)$ given in (2.6)-(2.9) the generalized Fibonacci hyperbolic cosine functions $(G F C F)$, the generalized Fibonacci hyperbolic sine functions $(G F S F)$, the generalized Lucas hyperbolic cosine functions $(G L C F)$, and the generalized Lucas hyperbolic sine functions $(G L S F)$, respectively.

According to the definitions in (2.6)-(2.9), we can say that GFCF and $G L C F$ are odd if $f(x)$ is odd or even. But if $f(x)$ is odd, GFSF and GLSF are even, and if $f(x)$ is even, GFSF and GLSF are odd. Note that we are unable to comment on the functions of $H S F$ and $H C F$ because they are also dependent to the additional functions $a(x)$ and $b(x)$. To able to do the investigation, it is a prerequisite that $f(x)$ must be even. For $a(x)=0$, some special cases can be investigated under the mentioned assumption. When $b(x)$ is odd, $\mathcal{H}_{s}(x)$ and $\mathcal{H}_{c}(x)$ is even and odd, respectively, and when $b(x)$ is even, $\mathcal{H}_{s}(x)$ and $\mathcal{H}_{c}(x)$ is odd and even, respectively.

According to Def. 2.1, we can give the following results.

Theorem 2.2. Let $x$ be any real number. Then, we have

$$
\mathcal{H}_{s}(x)=f(x) \mathcal{H}_{c}(x-1)+\mathcal{H}_{s}(x-2)
$$

and

$$
\mathcal{H}_{c}(x)=f(x) \mathcal{H}_{s}(x-1)+\mathcal{H}_{c}(x-2) .
$$

Proof. The Eqs. (2.10) and (2.11) can easily be showed by using Def. (2.1).

It is clear that the recurrence relations in (2.10) and (2.11) are in-homogeneous. Subtracting the $n$th and $(n+2)$ th terms of these sequences, we can give the new forth-order homogeneous forms

$$
\mathcal{H}_{s}(x+2)=\left(f^{2}(x)+2\right) \mathcal{H}_{s}(x)-\mathcal{H}_{s}(x-2)
$$

and

$$
\mathcal{H}_{c}(x+2)=\left(f^{2}(x)+2\right) \mathcal{H}_{c}(x)-\mathcal{H}_{c}(x-2) .
$$

Now we define the functions

$$
G_{s}(x, t)=\sum_{k=0}^{\infty} \mathcal{H}_{s}(x+k) t^{k}, G_{c}(x, t)=\sum_{k=0}^{\infty} \mathcal{H}_{c}(x+k) t^{k},
$$


and

$$
g_{s}(x, t)=\sum_{k=0}^{\infty} \mathcal{H}_{s}(x+k) \frac{t^{k}}{k !}, \text { and } g_{c}(x, t)=\sum_{k=0}^{\infty} \mathcal{H}_{c}(x+k) \frac{t^{k}}{k !},
$$

where $t$ is any real number. Note that generating functions are very useful tools to solve linear homogeneous recurrence relations with constant coefficients. Therefore, we write the following theorem.

Theorem 2.3 (Generating Function). The generating functions for HSF and HCF are

$$
G_{s}(x, t)=\frac{\mathcal{H}_{s}(x)+\mathcal{H}_{s}(x+1) t-\mathcal{H}_{s}(x-2) t^{2}-\mathcal{H}_{s}(x-1) t^{3}}{1-\left(f^{2}(x)+2\right) t^{2}+t^{4}}
$$

and

$$
G_{c}(x, t)=\frac{\mathcal{H}_{c}(x)+\mathcal{H}_{c}(x+1) t-\mathcal{H}_{c}(x-2) t^{2}-\mathcal{H}_{c}(x-1) t^{3}}{1-\left(f^{2}(x)+2\right) t^{2}+t^{4}} .
$$

Proof. Summing $G_{s}(x, t),-\left(f^{2}(x)+2\right) t^{2} G_{s}(x, t)$, and $t^{4} G_{s}(x, t)$ with some mathematical arrangements, the validity of Eq. (2.16) is seen. Repeating the same procedure for Eq. (2.17), the proof is completed.

We give the exponential generating function for $H S F$ and $H C F$ in the following theorem.

Theorem 2.4 (Exponential Generating Function). The exponential generating function for $H S F$ and $H C F$ are as follows:

$$
g_{s}(x, t)=\frac{A \alpha^{x} e^{\alpha t}-B \alpha^{-x} e^{-\beta t}}{\Delta}
$$

and

$$
g_{c}(x, t)=\frac{A \alpha^{x} e^{\alpha t}+B \alpha^{-x} e^{-\beta t}}{\Delta} .
$$

Proof. Considering MacLaurin expansion for the exponential function, we have

$$
\begin{aligned}
g_{s}(x, t)=\sum_{k=0}^{\infty} \mathcal{H}_{s}(x+k) \frac{t^{k}}{k !} & =\sum_{k=0}^{\infty} \frac{A \alpha^{x+k}-B \alpha^{-x-k}}{\Delta} \frac{t^{k}}{k !} \\
& =\frac{1}{\Delta}\left(A \alpha^{x} \sum_{k=0}^{\infty} \frac{(\alpha t)^{k}}{k !}-B \alpha^{-x} \sum_{k=0}^{\infty} \frac{\left(\alpha^{-1} t\right)^{k}}{k !}\right) \\
& =\frac{1}{\Delta}\left(A \alpha^{x} e^{\alpha t}-B \alpha^{-x} e^{\alpha^{-1} t}\right)
\end{aligned}
$$

which is the first claimed result. Other can be shown similarly.

\section{Elementary identities}

In this section, we present some elementary identities for the $H S F$ and $H C F$. To do this, we first start with the following three theorems, which will reduce to certain special cases. To decrease the size of the current paper, we will prove only one of them; because others can be proved similarly.

Theorem 3.1 (Vajda's identity). Let $x, y, z$, and $t$ be any real numbers. Then the HSF and HCF satisfy

$$
\begin{aligned}
\mathcal{H}_{s}(x+z) \mathcal{H}_{s}(y-t)-\mathcal{H}_{s}(x) & \mathcal{H}_{s}(y+z-t) \\
& =\frac{A B}{\Delta}\left(\mathcal{U}_{c}(x-y-z+t)-\mathcal{U}_{c}(x-y+z+t)\right)
\end{aligned}
$$


and

$$
\begin{aligned}
\mathcal{H}_{c}(x+z) \mathcal{H}_{c}(y-t)-\mathcal{H}_{c}(x) & \mathcal{H}_{c}(y+z-t) \\
& =-\frac{A B}{\Delta}\left(\mathcal{U}_{c}(x-y-z+t)-\mathcal{U}_{c}(x-y+z+t)\right),
\end{aligned}
$$

respectively.

Proof. Using the Binet's formula in (2.4), we can write the left-hand side as

$$
\begin{aligned}
\frac{A \alpha^{x+z}-B \alpha^{-(x+z)}}{\Delta} & \frac{A \alpha^{y-t}-B \alpha^{-(y-t)}}{\Delta}-\frac{A \alpha^{x}-B \alpha^{-x}}{\Delta} \frac{A \alpha^{y+z-t}-B \alpha^{-(y+z-t)}}{\Delta} \\
& =\frac{A B}{\Delta^{2}}\left[\alpha^{x-y-z+t}-\alpha^{x-y+z+t}+\alpha^{-(x-y-z+t)}-\alpha^{-(x-y+z+t)}\right] \\
& =\frac{A B}{\Delta}\left[\frac{\alpha^{x-y-z+t}+\alpha^{-(x-y-z+t)}}{\Delta}-\frac{\left.\alpha^{x-y+z+t}+\alpha^{-(x-y+z+t)}\right]}{\Delta}\right] \\
& =\frac{A B}{\Delta}\left[\mathfrak{U}_{c}(x-y-z+t)-\mathfrak{U}_{c}(x-y+z+t)\right],
\end{aligned}
$$

which is the first equation. Repeating the same procedure, the other can also be proved.

Theorem 3.2 (Vajda's identity of first kind). Let $x, y$, z, and $t$ be any real numbers. Then HSF and HCF satisfy

$$
\begin{aligned}
\mathcal{H}_{c}(x+z) \mathcal{H}_{s}(y-t)-\mathcal{H}_{c}(x) & \mathcal{H}_{s}(y+z-t) \\
& =\frac{A B}{\Delta}\left(\mathcal{U}_{s}(x-y-z+t)-\mathcal{U}_{s}(x-y+z+t)\right)
\end{aligned}
$$

and

$$
\begin{aligned}
\mathcal{H}_{s}(x+z) \mathcal{H}_{c}(y-t)-\mathcal{H}_{s}(x) & \mathcal{H}_{c}(y+z-t) \\
& =-\frac{A B}{\Delta}\left(\mathcal{U}_{s}(x-y-z+t)-\mathcal{U}_{s}(x-y+z+t)\right)
\end{aligned}
$$

respectively.

Theorem 3.3 (Vajda's identity of second kind). Let $x, y$, and $t$ be any real numbers. Then HSF and HCF satisfy

$$
\begin{aligned}
\mathcal{H}_{s}(x+z) \mathcal{H}_{s}(y-t)-\mathcal{H}_{c}(x) & \mathcal{H}_{c}(y+z-t) \\
& =-\frac{A B}{\Delta}\left(\mathcal{U}_{c}(x-y-z+t)+\mathcal{U}_{c}(x-y+z+t)\right)
\end{aligned}
$$

and

$$
\begin{aligned}
\mathcal{H}_{c}(x+z) \mathcal{H}_{c}(y-t)-\mathcal{H}_{s}(x) & \mathcal{H}_{s}(y+z-t) \\
& =\frac{A B}{\Delta}\left(\mathcal{U}_{c}(x-y-z+t)+\mathcal{U}_{c}(x-y+z+t)\right),
\end{aligned}
$$

respectively.

The results presented in Theorems 3.1-3.3 give the particular cases in the following.

Corollary 3.4 (d'Ocagne-type identity). For any real numbers $x$ and $y$, we have

$$
\begin{gathered}
\mathcal{H}_{s}(x+1) \mathcal{H}_{s}(y)-\mathcal{H}_{s}(x) \mathcal{H}_{s}(y+1)=-A B \frac{f(x)}{\Delta} \mathcal{U}_{s}(x-y), \\
\mathcal{H}_{c}(x+1) \mathcal{H}_{c}(y)-\mathcal{H}_{c}(x) \mathcal{H}_{c}(y+1)=A B \frac{f(x)}{\Delta} \mathcal{U}_{s}(x-y), \\
\mathcal{H}_{c}(x+1) \mathcal{H}_{s}(y)-\mathcal{H}_{c}(x) \mathcal{H}_{s}(y+1)=-A B \frac{f(x)}{\Delta} \mathcal{U}_{c}(x-y),
\end{gathered}
$$




$$
\begin{gathered}
\mathcal{H}_{s}(x+1) \mathcal{H}_{c}(y)-\mathcal{H}_{s}(x) \mathcal{H}_{c}(y+1)=A B \frac{f(x)}{\Delta} \mathcal{U}_{c}(x-y), \\
\mathcal{H}_{s}(x+1) \mathcal{H}_{s}(y)-\mathcal{H}_{c}(x) \mathcal{H}_{c}(y+1)=-A B \mathcal{U}_{c}(x-y),
\end{gathered}
$$

and

$$
\mathcal{H}_{c}(x+1) \mathcal{H}_{c}(y)-\mathcal{H}_{s}(x) \mathcal{H}_{s}(y+1)=A B \mathcal{U}_{c}(x-y) .
$$

Proof. Substituting $z=1$ and $t=0$ into Theorems 3.1-3.3, the proof is completed.

Corollary 3.5 (Catalan-type identity). For any real numbers $x$ and $y$, we have

$$
\begin{gathered}
\mathcal{H}_{s}(x+y) \mathcal{H}_{s}(x-y)-\left[\mathcal{H}_{s}(x)\right]^{2}=-A B\left[\mathcal{U}_{s}(y)\right]^{2}, \\
\mathcal{H}_{c}(x+y) \mathcal{H}_{c}(x-y)-\left[\mathcal{H}_{c}(x)\right]^{2}=A B\left[\mathcal{U}_{s}(y)\right]^{2}, \\
\mathcal{H}_{c}(x+y) \mathcal{H}_{s}(x-y)-\mathcal{H}_{c}(x) \mathcal{H}_{s}(x)=-A B \mathcal{U}_{s}(y) \mathcal{U}_{c}(y), \\
\mathcal{H}_{s}(x+y) \mathcal{H}_{c}(x-y)-\mathcal{H}_{s}(x) \mathcal{H}_{c}(x)=A B \mathcal{U}_{s}(y) \mathcal{U}_{c}(y), \\
\mathcal{H}_{s}(x+y) \mathcal{H}_{s}(x-y)-\left[\mathcal{H}_{c}(x)\right]^{2}=-A B\left[\mathcal{U}_{c}(y)\right]^{2},
\end{gathered}
$$

and

$$
\mathcal{H}_{c}(x+y) \mathcal{H}_{c}(x-y)-\left[\mathcal{H}_{s}(x)\right]^{2}=A B\left[\mathcal{U}_{c}(y)\right]^{2} .
$$

Proof. Substituting $z=y$ and $t=2 y-x$ into Theorems 3.1-3.3, the proof is completed.

Corollary 3.6 (Cassini-type identity). For any real number $x$, we have

$$
\begin{gathered}
\mathcal{H}_{s}(x+1) \mathcal{H}_{s}(x-1)-\left[\mathcal{H}_{s}(x)\right]^{2}=-A B\left[\frac{f(x)}{\Delta}\right]^{2}, \\
\mathcal{H}_{c}(x+1) \mathcal{H}_{c}(x-1)-\left[\mathcal{H}_{c}(x)\right]^{2}=A B\left[\frac{f(x)}{\Delta}\right]^{2}, \\
\mathcal{H}_{c}(x+1) \mathcal{H}_{s}(x-1)-\mathcal{H}_{c}(x) \mathcal{H}_{s}(x)=-A B \frac{f(x)}{\Delta}, \\
\mathcal{H}_{s}(x+1) \mathcal{H}_{c}(x-1)-\mathcal{H}_{s}(x) \mathcal{H}_{c}(x)=A B \frac{f(x)}{\Delta}, \\
\mathcal{H}_{s}(x+1) \mathcal{H}_{s}(x-1)-\left[\mathcal{H}_{c}(x)\right]^{2}=-A B,
\end{gathered}
$$

and

$$
\mathcal{H}_{c}(x+1) \mathcal{H}_{c}(x-1)-\left[\mathcal{H}_{s}(x)\right]^{2}=A B .
$$

Proof. Taking $t=z=1$ and $y=x$ in Theorems 3.1-3.3, the results follow.

Now we present the Honsberger formulas for $\mathcal{U}_{s}(x)$ and $\mathcal{U}_{c}(x)$ in the following.

Theorem 3.7 (Honsberger-type formula). Let $x$ and $y$ be any real numbers. Then,

$$
\begin{aligned}
\mathcal{H}_{s}(x+y) & =\mathcal{U}_{s}(x+1) \mathcal{H}_{c}(y)+\mathcal{U}_{c}(x) \mathcal{H}_{s}(y-1) \\
& =\mathcal{U}_{c}(x+1) \mathcal{H}_{s}(y)+\mathcal{U}_{s}(x) \mathcal{H}_{c}(y-1)
\end{aligned}
$$

and

$$
\begin{aligned}
\mathcal{H}_{c}(x+y) & =\mathcal{U}_{c}(x+1) \mathcal{H}_{c}(y)+\mathcal{U}_{s}(x) \mathcal{H}_{s}(y-1) \\
& =\mathcal{U}_{s}(x+1) \mathcal{H}_{s}(y)+\mathcal{U}_{c}(x) \mathcal{H}_{c}(y-1) .
\end{aligned}
$$


Proof. Using the Binet's formulas in (2.4) and (2.5), we can write

$$
\begin{aligned}
& U_{s}(x+1) H_{c}(y)+U_{c}(x) H_{s}(y-1)=\frac{\alpha^{x+1}-\alpha^{-x-1}}{\Delta} \frac{A \alpha^{y}+B \alpha^{-y}}{\Delta} \\
& +\frac{\alpha^{x}+\alpha^{-x}}{\Delta} \frac{A \alpha^{y-1}-B \alpha^{-y+1}}{\Delta} \\
& =\frac{1}{\Delta^{2}}\left(A \alpha^{x+y+1}-B \alpha^{-x-y-1}+A \alpha^{x+y-1}-B \alpha^{-x-y+1}\right) \\
& =\frac{1}{\Delta^{2}}\left(\alpha+\alpha^{-1}\right)\left(A \alpha^{x+y}-B \alpha^{-x-y}\right) \\
& =\frac{A \alpha^{x+y}-B \alpha^{-x-y}}{\Delta} \text {, }
\end{aligned}
$$

which is claimed result.

The next theorems present the well-known hyperbolic-like identities.

Theorem 3.8. For any real number $x$, we have

$$
\left[\mathcal{H}_{c}(x)\right]^{2}-\left[\mathcal{H}_{s}(x)\right]^{2}=\frac{4 A B}{\Delta^{2}} .
$$

Proof. Subtracting Eqs. (3.19) and (3.23) leads to the desired result.

Theorem 3.9. Let $x$ and $y$ be any real numbers. Then, HSF and HCF satisfy

$$
\frac{2}{\Delta} A B \mathcal{U}_{c}(x-y)=\mathcal{H}_{c}(x) \mathcal{H}_{c}(y)-\mathcal{H}_{s}(x) \mathcal{H}_{s}(y) .
$$

Proof. When $z=t=0$ in Eq. (3.6), the result follows.

Theorem 3.10 (De Moivre-type formula). Let $x$ be any real number. Then the following identities hold for any positive integer $n$ :

$$
\left[\mathcal{H}_{c}(x)+\mathcal{H}_{s}(x)\right]^{n}=\left(\frac{2 A}{\Delta}\right)^{n-1}\left[\mathcal{H}_{c}(n x)+\mathcal{H}_{s}(n x)\right]
$$

and

$$
\left[\mathcal{H}_{c}(x)-\mathcal{H}_{s}(x)\right]^{n}=\left(\frac{2 B}{\Delta}\right)^{n-1}\left[\mathcal{H}_{c}(n x)-\mathcal{H}_{s}(n x)\right] .
$$

Proof. We use the induction method to show the validity of the theorem. It is clear that Eq. (3.27) holds for $n=1$. Based on the assumption such that this equation is valid for any positive integer $k$, we can write

$$
\begin{aligned}
{\left[\mathcal{H}_{c}(x)+\right.} & \left.\mathcal{H}_{s}(x)\right]^{k+1}=\left[\mathcal{H}_{c}(x)+\mathcal{H}_{s}(x)\right]^{k}\left[\mathcal{H}_{c}(x)+\mathcal{H}_{s}(x)\right] \\
& =\left(\frac{2 A}{\Delta}\right)^{k-1}\left[\mathcal{H}_{c}(k x)+\mathcal{H}_{s}(k x)\right]\left[\frac{A \alpha^{x}+B \alpha^{-x}}{\Delta}+\frac{A \alpha^{x}-B \alpha^{-x}}{\Delta}\right] \\
& =\left(\frac{2 A}{\Delta}\right)^{k}\left[\frac{A \alpha^{k x}+B \alpha^{-k x}}{\Delta}+\frac{A \alpha^{k x}-B \alpha^{-k x}}{\Delta}\right] \alpha^{x} \\
& =\left(\frac{2 A}{\Delta}\right)^{k}\left[\frac{2 A \alpha^{(k+1) x}+B \alpha^{-(k+1) x}-B \alpha^{-(k+1) x}}{\Delta}\right] \\
& =\left(\frac{2 A}{\Delta}\right)^{k}\left[\mathcal{H}_{c}((k+1) x)+\mathcal{H}_{s}((k+1) x)\right],
\end{aligned}
$$

which completes the proof of Eq. (3.27). Repeating the same procedure, the other can be demonstrated. 


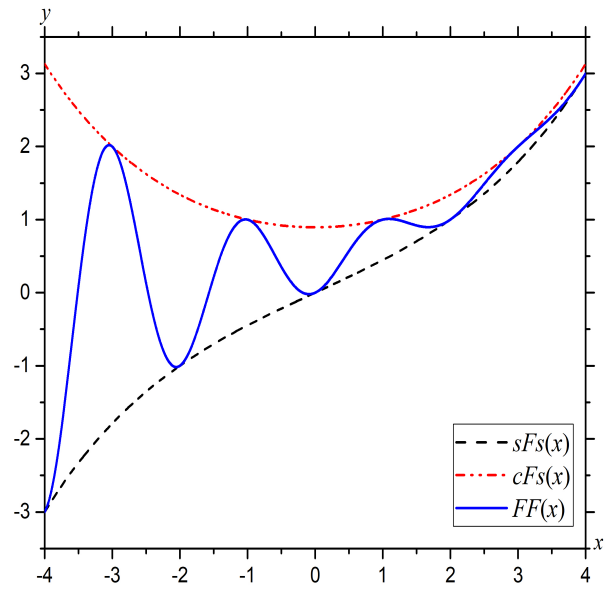

a. Quasi-sine and hyperbolic Fibonacci functions $[14,15]$

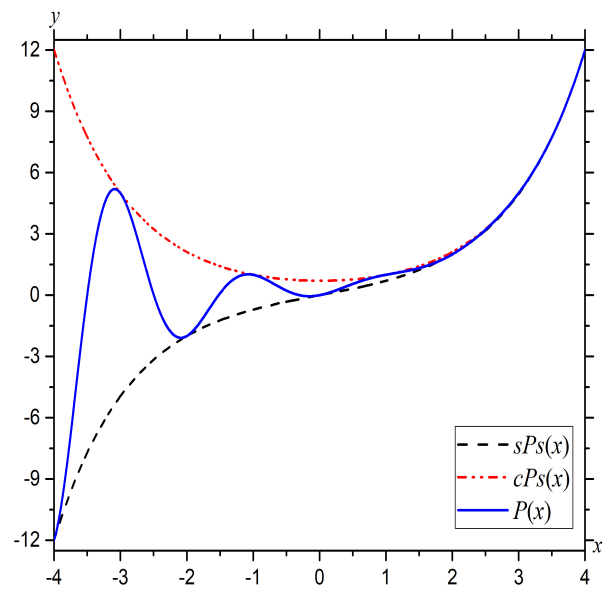

c. Quasi-sine and hyperbolic Pell functions [4]

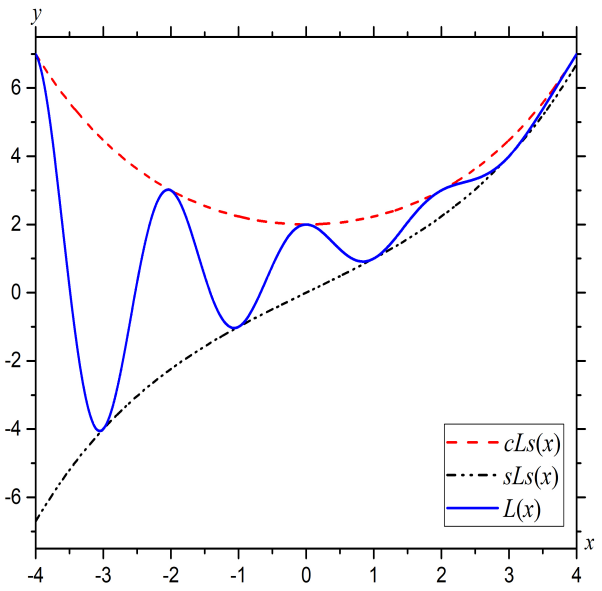

b. Quasi-sine and hyperbolic Lucas functions $[14,15]$

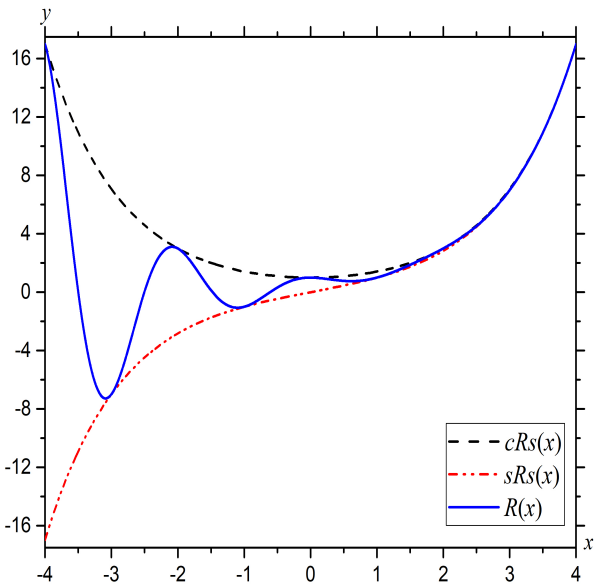

d. Quasi-sine and hyperbolic Modified Pell functions

Figure 1. Special forms of quasi-sine and Horadam hyperbolic functions

\section{Geometrical approach}

From Eqs. (2.4) and (2.5), we conclude that the functions $H S F$ and $H C F$ have the same form except for the sign of $\alpha^{-x}$. Hence, we can enter an auxiliary function that possesses 1 and -1 for consecutive integer values of $x$ into the Binet's formulas to guarantee continuous condition. To do this, the function $\cos (\pi x)$ may be the best choice. Consequently, the following definition arises naturally.

Definition 4.1. The quasi-sine Horadam function is defined as

$$
\mathcal{H}(a, b, f, x)=\mathcal{H}(x)=\frac{A \alpha^{x}-\cos (\pi x) B \alpha^{-x}}{\Delta} .
$$

Here, we can say that the definition in (4.1) can reduce to the quasi-sine forms of the special cases given in Table 1 depending on the choice of $a(x), b(x)$, and $f(x)$. In this case, we will omit the subscripts, such as $s$ and $c$, in the corresponding quantities.

Fig. 1 displays the distributions of special forms of quasi-sine and Horadam hyperbolic functions $\mathcal{H}(x)$ on the plane $O x y$. It should be noted that all the graphs given in this paper are designed in the ORIGINPRO ${ }^{\odot}$ using the numerical values obtained by an algorithm in the MATHEMATICA ${ }^{\odot}$. For validation purposes, we can consider the cases given in Figs. 

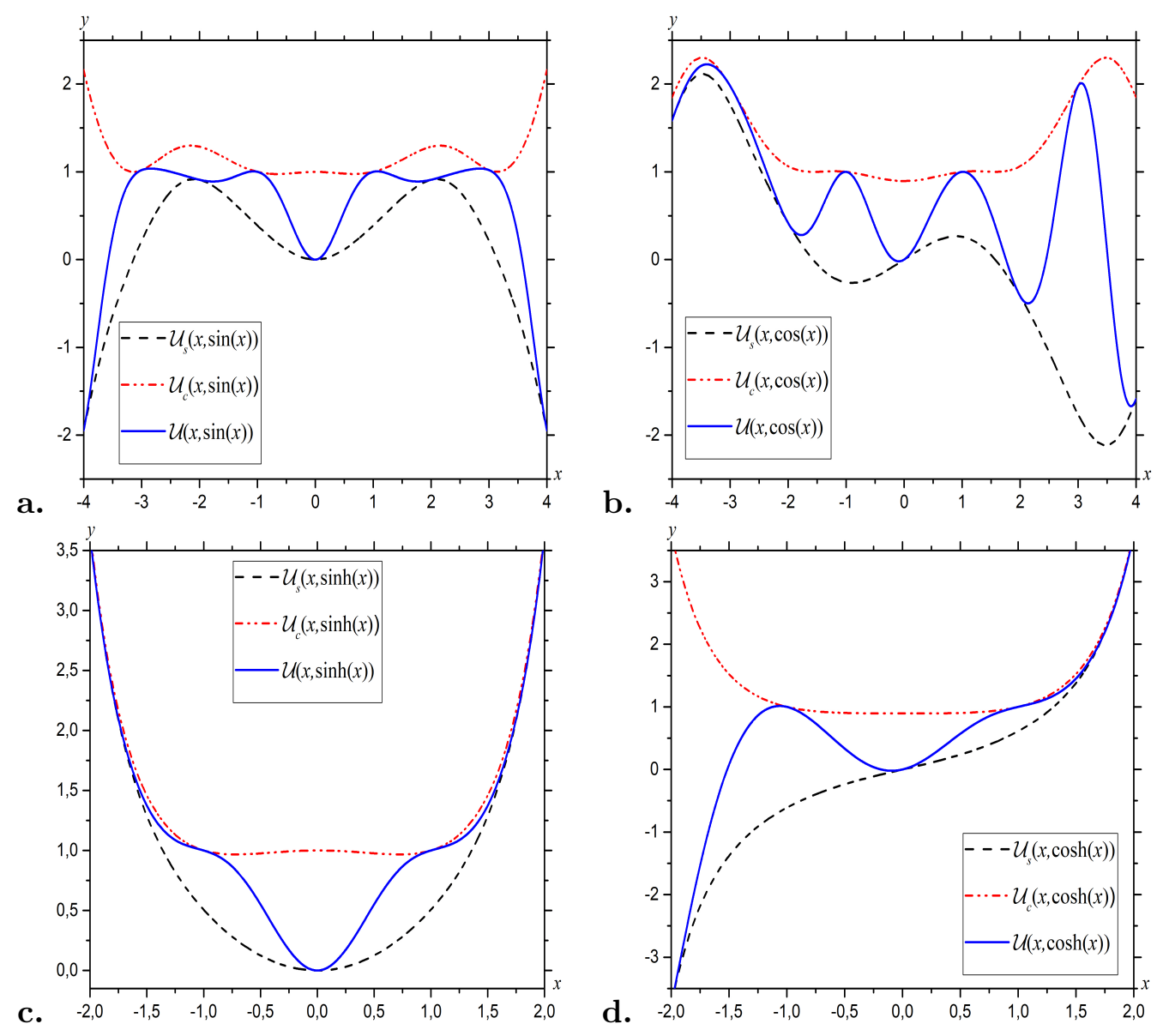

Figure 2. Variations of the generalized quasi-sine and hyperbolic Fibonacci functions on the plane Oxy: a. $f(x)=\sin (x)$, b. $f(x)=\cos (x)$, c. $f(x)=\sinh (x)$, d. $f(x)=\cosh (x)$

1a and 1c. In the mentioned cases, the distributions of the graphs must coincide with the ones given by Stakhov and Rozin [14,15], and Falcón and Plaza [4], respectively. The graphs show that this is indeed the case. According to Fig. 1, the graphs of the quasi-sine functions are wrapped by hyperbolic sine and cosine functions. For each sub-figure, the corresponding graphs have the same oscillating character in the qualitative sense. For the values of $x$ below a certain limit $x^{\star}$, the quasi-sine function $\mathcal{H}(x)$ reaches local extreme values. In many mechanical and physical problems, these are called the resonance values, denoted as $\mathcal{H}^{\star}(x)$. However, for a higher values above $x^{\star}$, the functions $\mathcal{H}(x)$ tend to the increase steadily. Note that the values of $x^{\star}$ in Fig. 1 differ for each function and can be determined from the graphs. We can conclude from the graphs that the parameters $a(x)$, $b(x)$, and $f(x)$ have a considerable influence on the values of $\mathcal{H}(x)$, not only quantitatively but also qualitatively.

Fig. 2 shows the variation of the generalized quasi-sine and hyperbolic Fibonacci functions on the plane $O x y$ for various choice of $f(x)$. As can be seen from the graphs, the function $\mathcal{U}_{c}(x)$ has a minimal value, however, the function $\mathcal{U}_{s}(x)$ has not the same character except for the choice of $f(x)=\sinh (x)$ (Fig. 2c).

Fig. 3 gives the opportunity to compare the influence of various choices of $a(x)$ and $b(x)$ for the same function $f(x)$. It follows from the graphs that the number of oscillations for constant integer values of $a(x)$ and $b(x)$ is more than when $a(x)$ and $b(x)$ are a non-constant function. 
a.

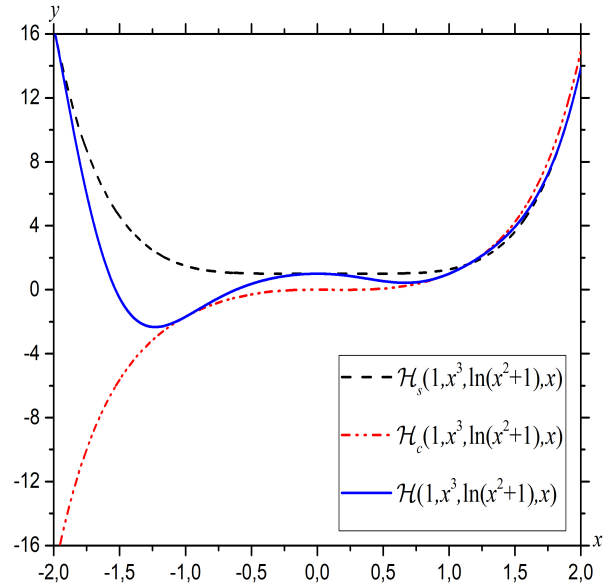

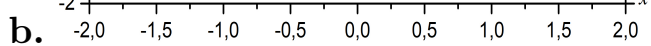

Figure 3. Variations of quasi-sine and Horadam hyperbolic functions (left) and the generalized quasi-sine and hyperbolic Fibonacci functions (right)

The common result from the graphs in Figs. 1, 2, and 3 is that, for integer values of $x$, the curve of the quasi-sine Horadam function is consecutively in contact with those of the Horadam hyperbolic functions.

According to Def. 4.1, we can give the following result.

Theorem 4.2. The following recurrence relation holds for any real number $x$,

$$
\mathcal{H}(x+2)=f(x) \mathcal{H}(x+1)+\mathcal{H}(x) .
$$

Proof. The proof can be completed from Eq. (4.1) directly.

Note that Eq. (4.2) is a second-order recurrence relation unlike Eqs. (2.10) and (2.11). We present the following theorem.

Theorem 4.3 (Vajda's identity). Let $r$ and $s$ be any integers and $x$ be a real number. Then,

$$
\begin{aligned}
\mathcal{H}(x+r) \mathcal{H}(x+s)- & \mathcal{H}(x) \mathcal{H}(x+r+s) \\
& =\frac{A B}{\Delta^{2}} \cos (\pi x)\left[\mathcal{V}(r+s)-(-1)^{s} \mathcal{V}(r-s)\right] .
\end{aligned}
$$


Proof. Considering trigonometric property such as $\cos (\pi(x+n))=(-1)^{n} \cos (\pi x)$ for all integer $n$, we can write

$$
\begin{aligned}
\mathcal{H}(x+r) \mathcal{H}(x+s)-\mathcal{H}(x) \mathcal{H}(x+r+s) & \frac{A \alpha^{x+r}-B \cos (\pi(x+r)) \alpha^{-(x+r)}}{\Delta} \frac{A \alpha^{x+s}-B \cos (\pi(x+s)) \alpha^{-(x+s)}}{\Delta} \\
= & -\frac{A \alpha^{x}-B \cos (\pi x) \alpha^{-x}}{\Delta} \frac{A \alpha^{x+r+s}-B \cos (\pi(x+r+s)) \alpha^{-(x+r+s)}}{\Delta} \\
= & \frac{A \alpha^{x+r}-(-1)^{r} B \cos (\pi x) \alpha^{-(x+r)}}{\Delta} \frac{A \alpha^{x+s}-(-1)^{s} B \cos (\pi x) \alpha^{-(x+s)}}{\Delta} \\
= & \quad-\frac{A \alpha^{x}-B \cos (\pi x) \alpha^{-x}}{\Delta} \frac{A \alpha^{x+r+s}-(-1)^{r+s} B \cos (\pi x) \alpha^{-(x+r+s)}}{\Delta} \\
= & \frac{A B}{\Delta^{2}} \cos (\pi x)\left[\alpha^{r+s}+\left(-1 \alpha^{x+r}(-1)^{s} B \cos (\pi x) \alpha^{-(x+s)}-(-1)^{r} B \cos (\pi x) \alpha^{-(x+r)} A \alpha^{x+s}\right]\right. \\
= & \frac{A B}{\Delta^{2}} \cos (\pi x)\left[\mathcal{V}(r+s)-(-1)^{r+s} B \cos (\pi x) \alpha^{-(x+r+s)}+B \cos (\pi x) \alpha^{-x} A \alpha^{x+r+s}\left(\alpha^{r-s}+(-1)^{r-s} \alpha^{-(r-s)}\right)\right],
\end{aligned}
$$

which is our claim.

From Vajda's identity, we also have the following special identities:

- For $x+r=y$ and $s=1$, we attain the d'Ocagne's identity:

$$
\mathcal{H}(x+1) \mathcal{H}(y)-\mathcal{H}(x) \mathcal{H}(y+1)=\frac{A B}{\Delta^{2}} \cos (\pi x)[\mathcal{V}(r+1)+\mathcal{V}(r-1)] .
$$

- For $r=-s$, we find the Catalan's identity:

$$
\mathcal{H}(x+r) \mathcal{H}(x-r)-\mathcal{H}(x)^{2}=-\frac{A B}{\Delta^{2}} \cos (\pi x)\left[(-1)^{r} \mathcal{V}(2 r)-2\right] .
$$

- For $r=-s=1$, we obtain the Cassini's identity:

$$
\mathcal{H}(x+1) \mathcal{H}(x-1)-\mathcal{H}(x)^{2}=A B \cos (\pi x) .
$$

We now introduce a new definition, which will be called Horadam spiral-like of hyperbolic type, in the following.

Definition 4.4. Three-dimensional Horadam spiral is defined as

$$
\mathcal{C H}(a, b, f, x)=\mathcal{C H} \mathcal{H}(x)=\frac{A \alpha^{x}-\cos (\pi x) B \alpha^{-x}}{\Delta}+i \frac{\sin (\pi x) B \alpha^{-x}}{\Delta} .
$$

It is clear that the real part of $\mathcal{E H}(a, b, f, x)$ is the quasi-sine Horadam function. The geometry of a Horadam spiral resembles to a three-dimensional spiral-like curve. Moreover, for integer values of $x$, its imaginary part equals to zero.

Figs. 4, 5, and 6 show the 3D graphs of the Horadam spirals for particular cases considered in Fig. 1, 2, and 3, respectively. It is concluded from the figures that the graphs does not have a constant curvature and torsion. Note that the cases in Figs. 4a and 4c were given by Stakhov and Rozin [15] and Falcón and Plaza [4].

Here, based on the assumption such that the $O y$ and $O z$ axes are respectively the real and imaginary axes, we construct the system of equations

$$
\left\{\begin{array}{l}
y(x)-\frac{A \alpha^{x}}{\Delta}=-\frac{\cos (\pi x) B \alpha^{-x}}{\Delta} \\
z(x)=\frac{\sin (\pi x) B \alpha^{-x}}{\Delta}
\end{array}\right.
$$




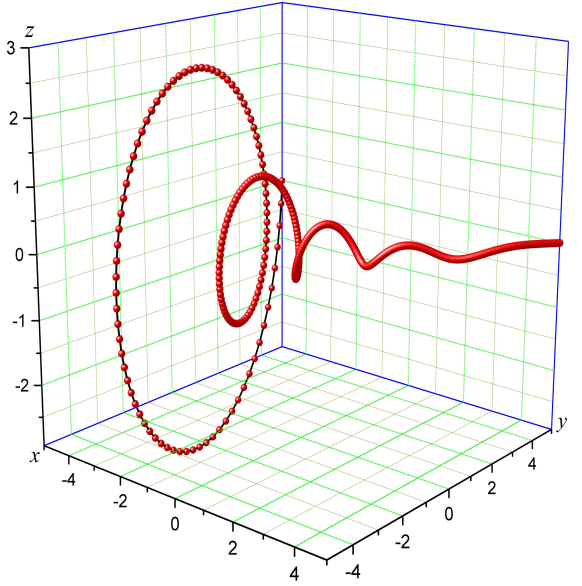

a. Fibonacci Spiral [15]

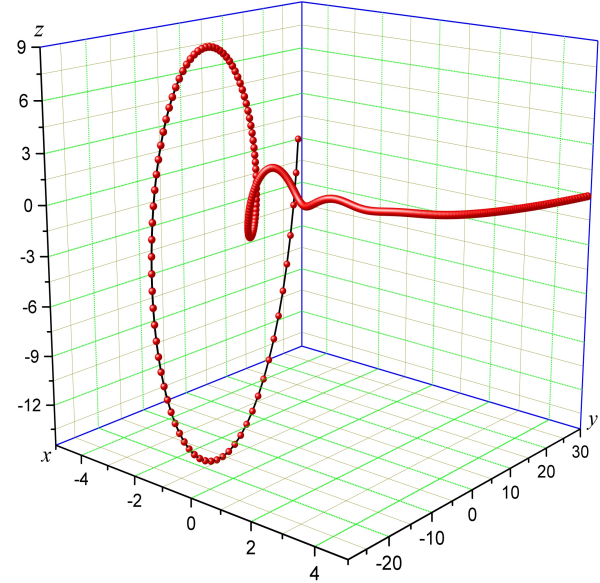

c. Pell Spiral [4]

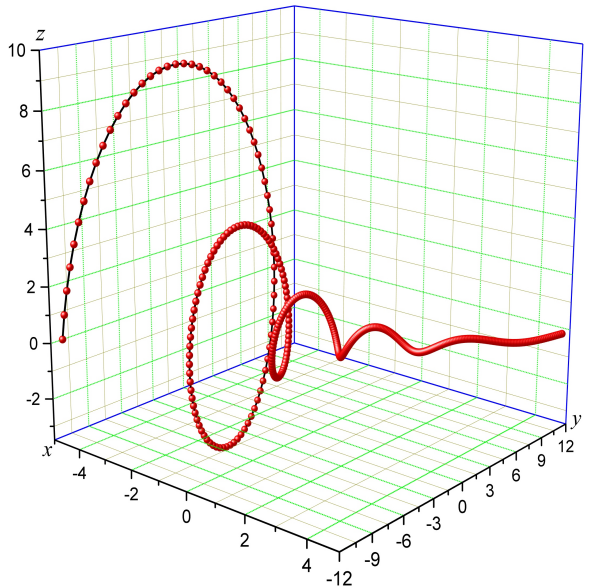

b. Lucas Spiral

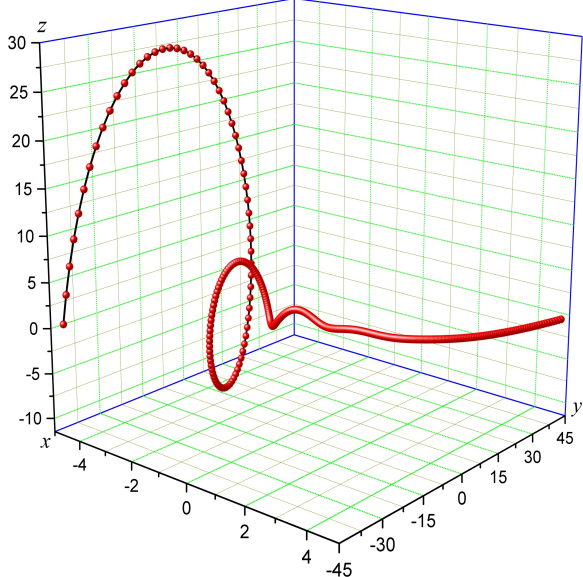

d. Modified Pell Spiral

Figure 4. Some special spirals

Adding all the equations obtained after taking square of both sides of each equation in (4.5), we have

$$
\left(y-\frac{A \alpha^{x}}{\Delta}\right)^{2}+z^{2}=\left(\frac{B \alpha^{-x}}{\Delta}\right)^{2}
$$

or equivalently

$$
z^{2}=\left(\mathcal{H}_{c}(x)-y\right)\left(y-\mathcal{H}_{s}(x)\right) .
$$

Note that we will call Eq. (4.6) the generalized Metallic Shofar. The reasons for giving this name was explained in references [15] and [4].

From Eq. (4.4), we have the following result.

Theorem 4.5. The following recurrence relation holds for any real number $x$,

$$
\mathcal{E H}(x+2)=f(x) \mathcal{E H}(x+1)+\mathcal{E} \mathcal{H}(x) .
$$




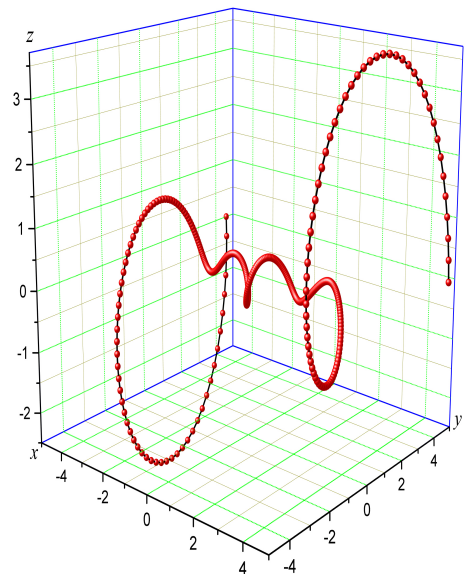

a.

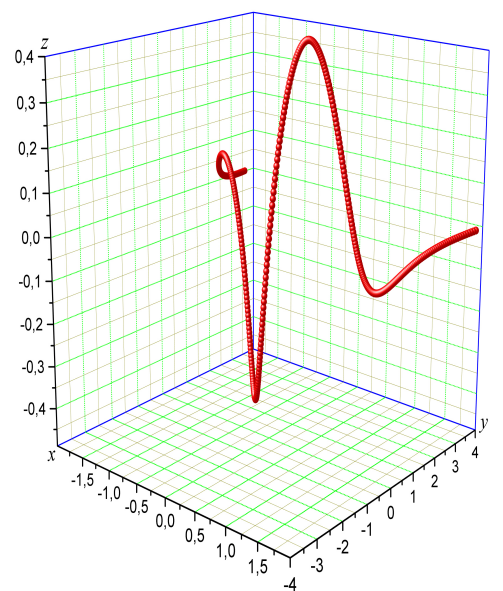

c.

Figure 5. Some generalized Fibonacci spirals: a. $f(x)=\sin (x)$, b. $f(x)=$ $\cos (x)$, c. $f(x)=\sinh (x)$, d. $f(x)=\cosh (x)$

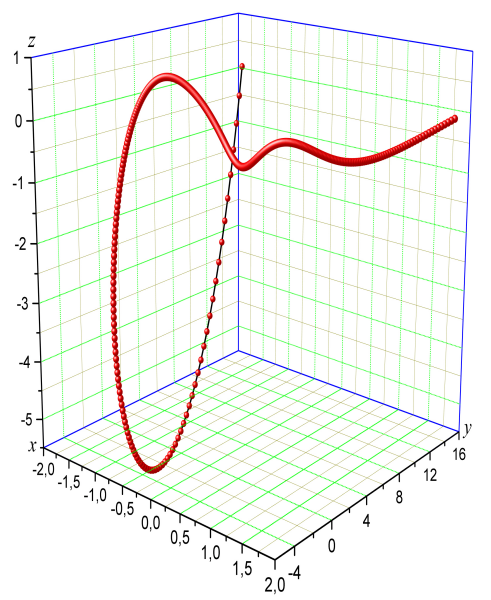

a.

b.

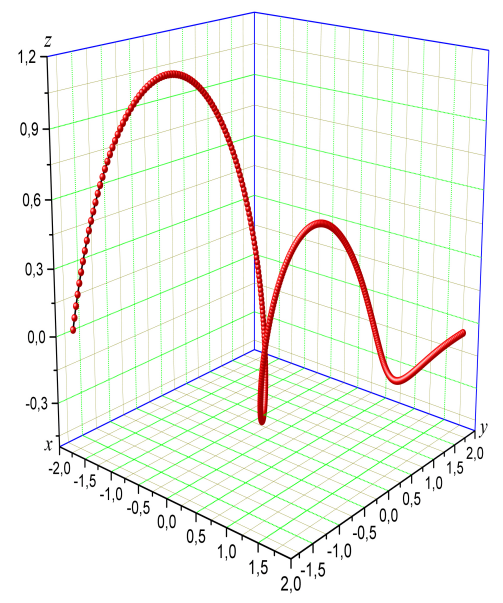

Figure 6. Some Horadam spirals for $f(x)=\ln \left(x^{2}+1\right)$ : a. $a(x)=1$ and $b(x)=x^{3}$, b. $a(x)=0$ and $b(x)=1$

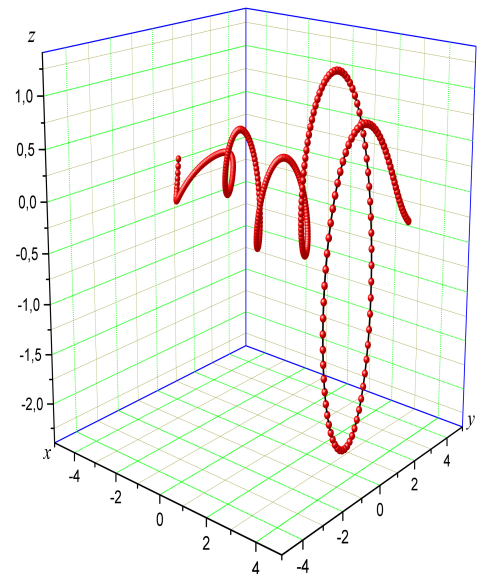

b.

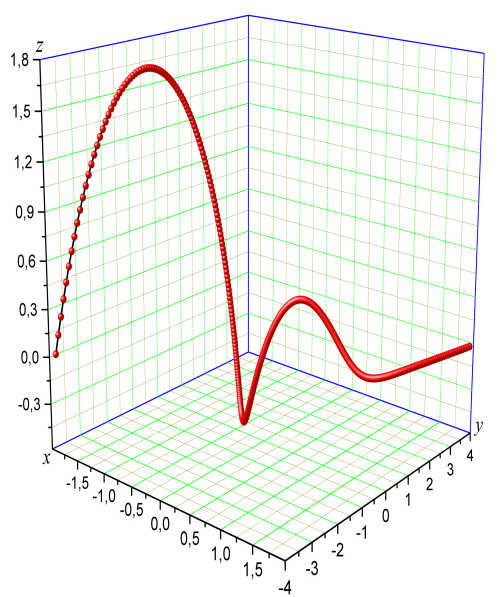

d.

$$
\text { . }
$$




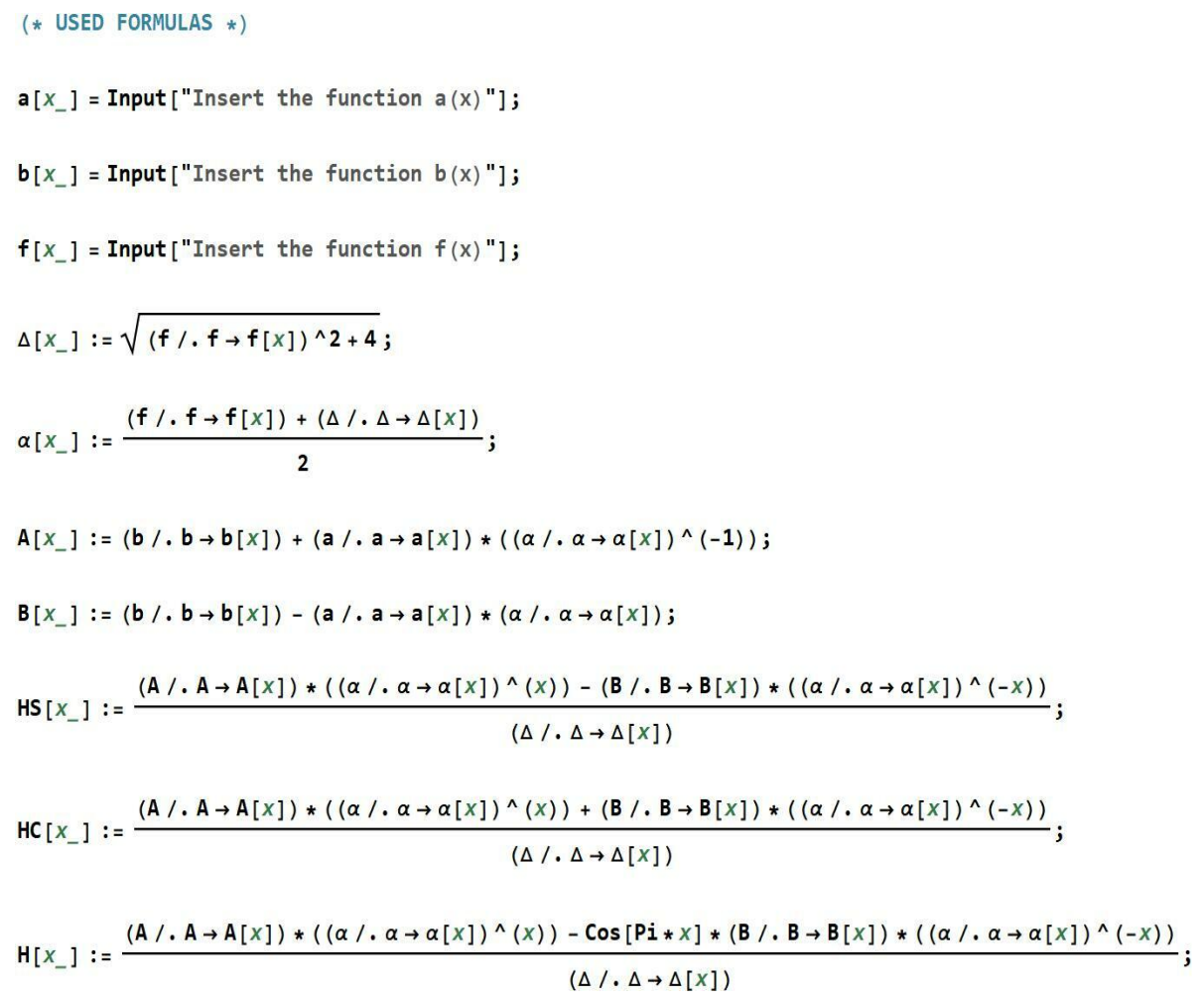

Figure 7. Algorithm for the Horadam functions

Proof. From the properties such as $\cos (\pi(x+n))=(-1)^{n} \cos (\pi x)$ for all integer $n$ and $\sin (\pi(x+n))=(-1)^{n} \sin (\pi x)$ for all integer $n$, the claimed result can be proved.

The next theorem displays Vajda's identity for the three-dimensional Horadam spiral.

Theorem 4.6 (Vajda's identity). Let $r$ and $s$ be any integers and $x$ be a real number. Then we have

$$
\begin{aligned}
\mathcal{E H}(x+r) \mathcal{C H}(x+s)-\mathcal{C H}(x) \mathcal{E} \mathcal{H}(x+r+s) \\
\quad=\frac{A B}{\Delta^{2}} \cos (\pi x)\left[\mathcal{V}(r+s)-(-1)^{s} \mathcal{V}(r-s)\right] .
\end{aligned}
$$

Proof. Another way to represent Eq. (4.4) is as follows:

$$
\mathcal{E H}(x)=\frac{A \alpha^{x}+i e^{i \pi\left(\frac{1}{2}-x\right)} B \alpha^{-x}}{\Delta} .
$$

Applying the same procedure in the proof of Theorem 4.3 with some additional operations, the result follows.

There is a surprising result here. The right-hand side of Eqs. (4.3) and (4.9) are the same; even though the left side of Eq. (4.9) is composed of complex functions. Hence, we conclude that right-hand sides of all the particular cases given for the quasi-sine Horadam functions are valid for those of the Horadam spiral. We list them below.

- When $x+r=y$ and $s=1$, the d'Ocagne's identity is obtained:

$\mathcal{E H}(x+1) \mathcal{C H} \mathcal{H}(y)-\mathcal{C H}(x) \mathcal{C H}(y+1)=\frac{A B}{\Delta^{2}} \cos (\pi x)[\mathcal{V}(r+1)+\mathcal{V}(r-1)]$.

- For $r=-s$, the Catalan's identity is found:

$$
\mathcal{C H}(x+r) \mathcal{E} \mathcal{H}(x-r)-\mathcal{C H}(x)^{2}=-\frac{A B}{\Delta^{2}} \cos (\pi x)\left[(-1)^{r} \mathcal{V}(2 r)-2\right] .
$$


- If $r=-s=1$, the Cassini's identity is as follows:

$$
\mathcal{E H}(x+1) \mathcal{C H}(x-1)-\mathcal{e} \mathcal{H}(x)^{2}=A B \cos (\pi x) .
$$

\section{PC algorithm}

According to the definitions given in (2.4)-(2.9), (4.1), and (4.4), there are three functions of real numbers $x$, i.e. $a(x), b(x)$, and $f(x)$, and their various combinations, i.e. $A(x), B(x), \alpha(x)$, and $\Delta(x)$. Due to the mentioned and other additional reasons, it can be very tedious to compute the results presented in this paper sometimes. To eliminate this difficulty, we give a PC algorithm composed in version 11.0 of the MATHEMATICA ${ }^{\odot}$. It should be noted that it may be not running for older versions. Here, we have big handicaps to construct the program since the above-mentioned functions can be chosen arbitrarily, provided that they are continuous. Hence, we apply a different approach to accomplish it. In the algorithm, we denote $\mathcal{H}_{s}(x), \mathcal{H}_{c}(x)$, and $\mathcal{H}(x)$ by $\mathrm{HS}(x), \mathrm{HC}(x)$, and $\mathrm{H}(x)$ respectively.

Fig. 7 shows the PC algorithm developed for the quasi-sine and Horadam hyperbolic functions. It is sufficient to run it once after writing all definitions in a cell; however, it should not be forgotten that the first letter in functions must be capital. The program will keep the definitions of $\mathcal{H}_{s}(x), \mathcal{H}_{c}(x)$, and $\mathcal{H}(x)$ in its memory. After this, a formula to be computed are entered in a new cell and it can be computed easily.

Acknowledgment. This work was financially supported by the Research Fund of Kastamonu University via project number KÜBAP-01/2018-77.

\section{References}

[1] H. Bulut, Y. Pandir, and H.M. Baskonus, Symmetrical hyperbolic Fibonacci function solutions of generalized Fisher equation with fractional order, AIP Conf. Proc. 1558 (1), 1914-1918, 2013.

[2] S.M. Ege and E. Misirli, The modified Kudryashov method for solving some fractionalorder nonlinear equations, Adv. Differ. Equ. 2014, Art. No. 135, 2014.

[3] S. Falcón and Á. Plaza, On the Fibonacci k-numbers, Chaos Solitons Fractals, 32 (5), 1615-1624, 2007.

[4] S. Falcón and Á. Plaza, The k-Fibonacci hyperbolic functions, Chaos Solitons Fractals, 38 (2), 409-420, 2008.

[5] A.F. Horadam, A generalized Fibonacci sequence, Amer. Math. Monthly, 68 (5), 455459, 1961.

[6] A.F. Horadam, Generating functions for powers of a certain generalized sequence of numbers, Duke. Math. J. 32 (3), 437-446, 1965.

[7] A.F. Horadam, Basic properties of a certain generalized sequence of numbers, Fibonacci Quart. 3 (3), 161-176, 1965.

[8] A.F. Horadam, Special properties of the sequence $W_{n}(a, b ; p, q)$, Fibonacci Quart. 5 (3), 424-434, 1967.

[9] T. Koshy, Fibonacci and Lucas Numbers with Applications, John Wiley and Sons, Inc., Hoboken, NJ, 2019.

[10] Y. Pandir, Y. Gurefe, and E. Misirli, A new approach to Kudryashov's method for solving some nonlinear physical models, Int. J. Phys. Sci. 7 (21), 2860-2866, 2012.

[11] S.S. Ray, New analytical exact solutions of time fractional KdV-KZK equation by Kudryashov methods, Chinese Physics B. 25 (4), 040204, 2016.

[12] A.P. Stakhov, Hyperbolic Fibonacci and Lucas functions: a new mathematics for the living nature, ITI, Vinnitsa, 2003. 
[13] A.P. Stakhov and S. Aranson, Hyperbolic Fibonacci and Lucas functions, "Golden" Fibonacci goniometry, Bodnar's geometry, and Hilbert's fourth problem, Appl. Math. 2, 74-84, 2011.

[14] A.P. Stakhov and B. Rozin, On a new class of hyperbolic functions, Chaos Solitons Fractals, 23, 379-389, 2005.

[15] A.P. Stakhov and B. Rozin, The Golden Shofar, Chaos Solitons Fractals, 26 (3), 677-684, 2005.

[16] A.P. Stakhov and B. Rozin, The "golden" hyperbolic models of Universe, Chaos Solitons Fractals, 34 (2), 159-171, 2007.

[17] A.P. Stakhov and I.S. Tkachenko, Hyperbolic Fibonacci trigonometry, Rep. Ukr. Acad. Sci. 208, 9-14, 1993. 